\title{
The otoferlin interactome in neurosensory hair cells: Significance for synaptic vesicle release and trans-Golgi network (Review)
}

\author{
MAGDALENA ŻAK ${ }^{1}$, MARKUS PFISTER $^{2}$ and NIKOLAUS BLIN ${ }^{1}$ \\ ${ }^{1}$ Division of Molecular Genetics, Institute of Human Genetics, University of Tübingen, 72074 Tübingen; \\ ${ }^{2}$ Department of Otorhinolaryngology, University of Tübingen, THRC, Molecular Genetics, 72076 Tübingen, Germany
}

Received April 11, 2011; Accepted May 9, 2011

DOI: $10.3892 /$ ijmm.2011.716

\begin{abstract}
Sound perception in terrestrial vertebrates relies on a structure in the inner ear consisting of the utriculus, sacculus and lagena. In mammals, the lagena has developed into the cochlea where mechanotransduction at ciliated cells leads to ion influx via regulated ion channels. To maintain proper $\mathrm{Ca}^{2+}$ concentration many cellular systems use a variety of functional proteins; the neurosensory systems use calcium-sensors like hippocalcin, visinin or recoverin. In cochlear hair cells the $230 \mathrm{kDa}$ protein otoferlin has been suggested to play this role. While several observations support this hypothesis additional data argue for a more expanded functional profile of otoferlin. Evidence for otoferlin's multiple roles and newer results on otoferlin's interacting partners are presented and the existence of a protein complex as a functional unit ('interactome') in the cochlea and further tissues is suggested.
\end{abstract}

\section{Contents}

1. Introduction

2. Calcium sensors

3. Otoferlin as a calcium sensor

4. Otoferlin's interactome

\section{Introduction}

Two neurosensory functions aid in the orientation of mobile terrestrial animals and allow perception over larger distances: the visual and auditory senses. Humans register sound waves in the range of $20-20,000 \mathrm{~Hz}$, while other vertebrates show

Correspondence to: Dr Magdalena Żak, Division of Molecular Genetics, Institute of Human Genetics, University of Tübingen, Wilhelmstr 27, 72074 Tübingen, Germany

E-mail: magdalena.zak1@googlemail.com

Key words: neurosensory systems, ciliated cells, calcium sensor, synaptic ribbons, exocytosis, endocytosis capacities below or above the human limits. The impressive sensitivity of hearing is derived from the transformation of mechanical signals to ion flux and depolarization resulting in electric signals transmitted to the brain via the eighth nerve. This complex process requires a highly specialized structure and the mammalian cochlea is the result of evolution of a refined instrument for sound perception (1). One key element of the intricate cochlear organ is a set of ciliated cells (inner and outer hair cells) where mechanical movement of the cilia opens or closes cation channels (2). Since the cilia reach into a space filled with endolymph rich with potassium, channel opening leads to potassium influx and depolarization of hair cells. This event, in turn, opens calcium channels, thus leading to further depolarization by allowing calcium influx, which triggers synaptic vesicle exocytosis and neurotransmitter release $(3,4)$. To distinguish temporally between sounds these steps must occur quickly; thus, to facilitate high rates of sustained synaptic transmission inner hair cells contain specialised structures, the so-called synaptic ribbons. These are capable of tethering numerous synaptic vesicles at release sites (5) for a speedy, coordinated process. Moreover, calcium-activated potassium channels control a rapid potassium flux required for improved perception of high frequencies (6). This short description underlines the significance of cations and their channels in the hearing process.

\section{Calcium sensors}

In the many specialised cell types of the body numerous proteins functioning as calcium sensors have been reported (7-12). In neurons of the brain and in neurosensory systems proteins like hippocalcin (13) or visinin and recoverin (14) play a crucial role as key mediators of many cellular functions including synaptic plasticity or regulation of ion channels. In the ciliary membranes, the sites of odorant transduction, for example, the guanylyl cyclase activating protein 1 (GCAP1), responds to the free $\mathrm{Ca}^{2+}$ by inhibiting or stimulating further proteins, some of those also linked to phototransduction. This demonstrates that a single $\mathrm{Ca}^{2+}$ sensor, working in two opposite modes of signalling, can serve two sensory processes (olfactory and visual) (15).

Acoustic perception is a highly complex sense and one key component, the cochlea of the inner ear, consist of many specialised cell types. Therefore, not surprisingly, more than 


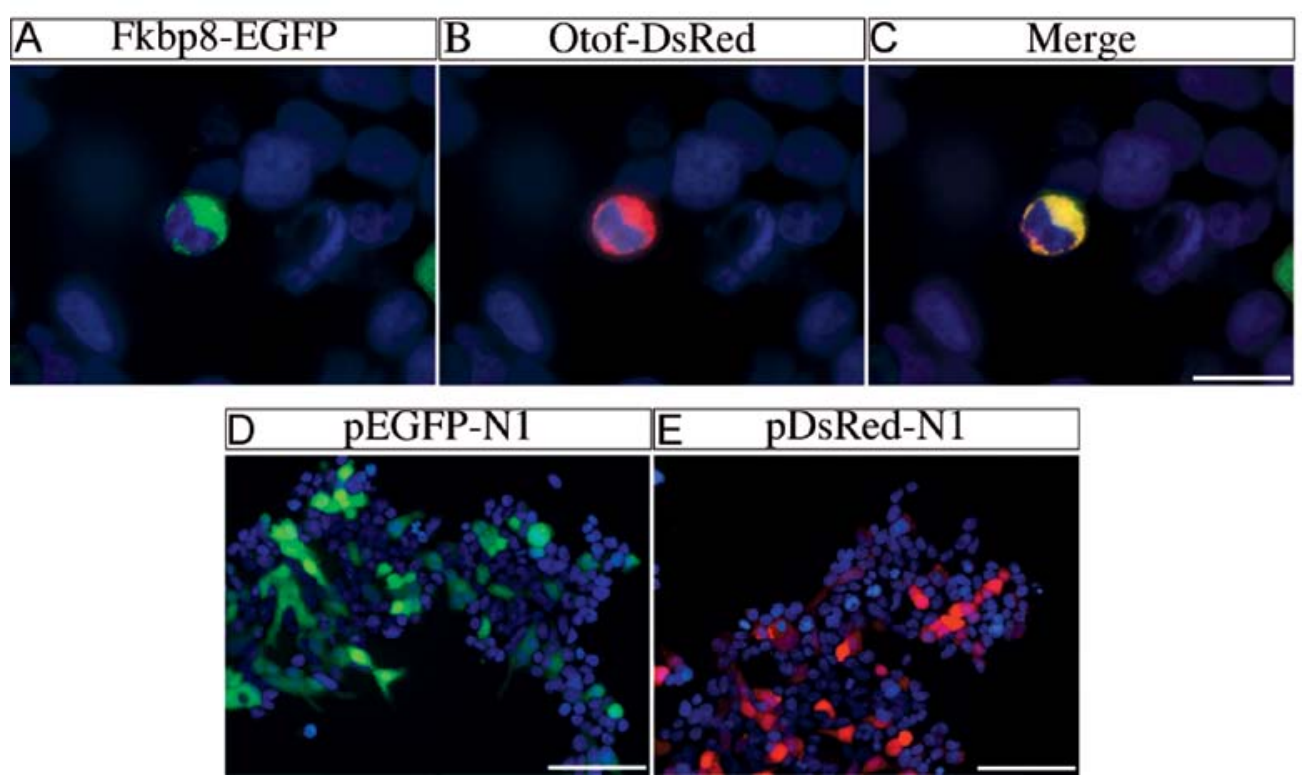

Figure 1. Co-localization of Fkbp8 and otoferlin in HEK293 cells. HEK293 cells were simultaneously transfected with (A) Fkbp8-pEGFP and (B) otoferlinpDsRed. (C) The resulting mixed colour (yellow) signal of co-localization was observed upon merging of A and B photographs. (D and E) Transfection with empty pEGFP-N1 and pDsRed-N1 leads to a ubiquitous distribution of fluorescent signals in cells. Cell nuclei were counterstained with DAPI. Scale bars, $20 \mu \mathrm{m}$.

200 genes are expected to participate in its structural build-up and function (data from http://hereditaryhearingloss.org/). A number of proteins coded by these genes cooperate in a temporal and spacial setting and elucidation of such interacting complexes, called 'interactomes', has moved into the focus of recent research. For one syndromic hearing defect (Usher syndrome type I) the cooperation of myosin VIIa, harmonin, sans, protocadherin 15 and cadherin 23 was shown to be essentail for the organisation of sensory hair cells (16). In the non-syndromic hearing impairment DFNA4, where the primary target of mutation was found to be MYH14 $(17,18)$, actin filaments interact with 2E4-Kaptin and in stereocilia myosin XVa together with whirlin control the development of these cells $(19,20)$.

\section{Otoferlin as a calcium sensor}

In the auditory system the neurons were reported to contain their own calcium sensors, synaptotagmin I/II (21), that mediate neurotransmitter release in a complex termed SNARE (22). In the inner ear, cochlear and vestibular hair cells may have their sensor protein: otoferlin. This suggestion was based on several observations. (i) Mature hair cells do not show significant amounts of synaptotagmin (23); (ii) synaptotagmin has two $\mathrm{Ca}^{2+}$ binding domains $(\mathrm{C} 2 \mathrm{~A}, \mathrm{C} 2 \mathrm{~B})$ and otoferlin displays six $\mathrm{C} 2$ domains $(\mathrm{C} 2 \mathrm{~A}-\mathrm{C} 2 \mathrm{~F})(24,25)$. Though the amino acid alignment indicates lack of complete conservation within the OTOF C2 motifs, dysferlin, while also binding $\mathrm{Ca}^{2+}$, deviates from the classic C2 core motif as well (26); (iii) otoferlindeficient mice are deaf but show functional mechanoelectrical transduction. Their hair cells do not release neurotransmitters in response to calcium influx (27); (iv) in hair cells, replenishment of synaptic vesicles was demonstrated to be otoferlin-dependent (28); and (v) five of the six C2 domains of otoferlin stimulate membrane fusion in a calcium-dependent fashion (29).
Table I. Potential interacting partners of otoferlin identified by yeast-2-hybrid system.

\begin{tabular}{lc}
\hline Protein & Cochlea cDNA \\
\hline Fkbp8 & + \\
cAMP & + \\
Col2a1 & + \\
RhoGTPase3 & + \\
MAP kinase2 & + \\
Clusterin & + \\
Pleiotrophin & + \\
Rps21 & + \\
Igfbp2 & + \\
ALG-2 & + \\
\hline
\end{tabular}

Expression of this set of candidates was confirmed by RT-PCR in cochlear RNA extracts (33).

\section{Otoferlin's interactome}

In addition to otoferlin's contribution to calcium-dependent exocytosis via 'ribbon synapses', as discussed above, several new data suggest otoferlin's participation in the endosomal trans-Golgi network. While these observations do not exclude otoferlin as a calcium sensor involved in membrane fusion reactions, they may extend its action potential to vesicle recycling/membrane trafficking. One first indication of such additional functions was the demonstration of its expression in a widened pattern, including the brain (25), the vestibular system, neurons and nerve fibers. Also, apart from its presence in cochlear inner hair cells, otoferlin was found to be present in mature outer hair cells responsible for low-frequency 


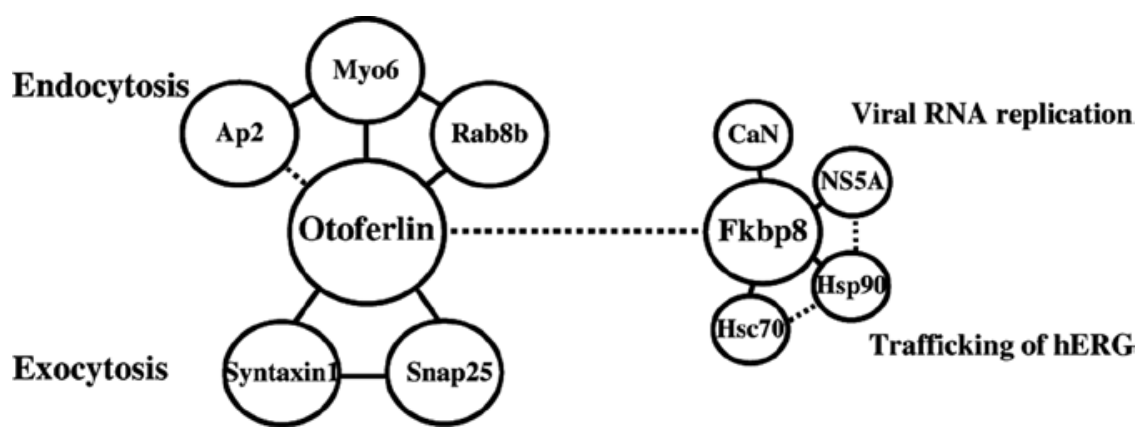

Figure 2. Schematic diagram of otoferlin interactom. Schematic interactom of otoferlin comprises verified (solid line) and hypothetical (broken line) binding partners involved in exocytosis via ribbon synapses (Syntaxin 1, Snap25) and endosomal trans-Golgi trafficking (Myo6, Rab8b, Ap2). The diagram includes Fkbp8, a further potential interactor suggested by yeast-2-hybrid screening, and a set of known binding proteins of Fkbp8 [calcineurin (CaN), HCV nonstructural protein 5A (NS5A), Hsp90 and Hsc70].

processing (30). Its subcellular distribution beyond regions of synaptic vesicle fusion and its association with Golgi markers suggested a more ubiquitous role. Protein-protein association studies demonstrated that otoferlin can not only interact with syntaxin 1 and Snap25, both ribbon synaptic components (27) but with Rab8 and Myo6, two proteins involved in the transGolgi network and in cargo sorting $(31,32)$.

Screening for otoferlin's partners using the yeast-2-hybrid system suggested further candidates (33) (Table I). Fkbp8 is a multifunctional protein shown to be involved in patterning of neuronal tissues (34). Since it displays a transmembrane domain it is expected to be localized at such. In fact, it was shown to be associated with the endoplasmic reticulum and the mitochondria $(35,36)$. Additionally, Fkbp8 is an interacting partner of two other membrane-anchored proteins: $\mathrm{Bcl}-2$ and $\mathrm{Bcl}-\mathrm{xL}$, which are mitochondria-bound anti-apoptotic molecules $(36,37)$. Fkbp8 is required to antagonize Sonic Hedgehog signaling during formation of the central nervous system and together with Hsp73 and Hsp90 regulates trafficking of hERG potassium channels in the heart $(34,38)$. Moreover, it interacts with the NS5A protein of the hepatitis $\mathrm{C}$ virus and recruits Hsp90 to form a complex participating in viral RNA replication (39). Fkbp8 has presently been investigated in more detail and was co-localized with otoferlin in HEK cells (Fig. 1) but not in cochlear hair cells, the site of otoferlin's presence. Fkbp8 displays an interesting expression pattern during cochlear maturation in rodents, i.e. from the time of pre-hearing onset (P5, postnatal day 5) its low level expression increases and peaks at P10 and decreases again after hearing onset (>P15). At the same time, its expression pattern is broadened: in addition to spiral ganglia it appears within cells of the stria vascularis (unpublished data). While these findings assign some role to Fkbp8 in the maturation of rodent hearing, it appears not to be a definite partner in the cochlear otoferlin interactome.

Recent data suggest a further component of this interactome. This candidate, Ap2, is a member of the clathrin-dependent endocytosis complex and is itself a heterotetramere which is called adaptor protein complex 2 (40). Ap2 was demonstrated to interact with Myo6 in intestinal epithelia (41) and otoferlin to bind Myo6 in cochlea (31) suggesting otoferlin's possible complex formation with Ap2 via Myo6. This model was most recently supported by co-localization studies in rodent cochlea.
Also, both genes are activated within the same time frame in the rodent brain (42). These data additionally support the idea of otoferlin contributing to endocytosis in auditory hair cells.

In a new model of otoferlin impairment, only one amino acid in a $\mathrm{C} 2$ domain was mutated. In these mice, the overall otoferlin structure does not differ significantly from the wildtype protein and the structure of the synapses remains the same. Interaction with $\mathrm{Ca}^{2+}$ and synaptic vesicles are expected not to be affected (28). Thus, the authors' explanation aims at a disturbed protein-protein interaction in otoferlin's interactome.

In summary, all these findings suggest that otoferlin executes its function in possibly variable cooperation complexes depending on whether action at ribbon synapses, endocytic or secretory trafficking is required (Fig. 2). Synaptic transmission involving otoferlin as a calcium-sensor has been reported (29) but for neurotransmitter release in hair cells it may not be entirely necessary: hypothyroid rats that lack otoferlin in their IHCs show IHC exocytosis (43). Whether this suggests that an otoferlin interactome may be substituted by a comparable protein complex without otoferlin remains to be elucidated in further investigations. Such studies should show whether a different, yet uncharacterized protein replaces otoferlin's function or whether the otoferlin interactome may only be required for a defined time span during cochlear maturation. Despite such open questions it is clear that otoferlin plays a crucial role in the inner ear's function with mutations resulting in auditory neuropathy (44).

\section{Acknowledgements}

The authors are grateful to Dr Paulina Heidrych and Dr Susanne Duncker for communicating some of their data from their dissertations and Dr Marlies Knipper for numerous discussions. The project was supported by the Landesgraduiertenstipendium Baden-Württemberg to M.Ż. and Honorary Humboldt Fellowship to N.B. The authors are grateful to Masaki Nishimura, Shiga University of Medical Science, Japan for the Fkbp8-EGFP construct.

\section{References}

1. Grothe B, Pecka M and McAlpine D: Mechanisms of sound localization in mammals. Physiol Rev 90: 983-1012, 2010. 
2. Ashmore J, Avan P, Brownell WE, et al: The remarkable cochlear amplifier. Hear Res 266: 1-17, 2010.

3. Chan DK and Hudspeth AJ: $\mathrm{Ca}^{2+}$ current-driven nonlinear amplification by the mammalian cochlea in vitro. Nat Neurosci 8: 149-155, 2005.

4. Glowatzki E and Fuchs PA: Transmitter release at the hair cell ribbon synapse. Nat Neurosci 5: 147-154, 2002.

5. Liberman D, Herskovitz T and Alroy G: Oral disodium cromoglycate in the treatment of systemic mastocytosis. Harefuah 99: 431-432, 1980 (In Hebrew).

6. Fuchs PA and Evans MG: Potassium currents in hair cells isolated from the cochlea of the chick. J Physiol 429: 529-551, 1990.

7. Brown EM, Chen CJ, Kifor O, et al: Ca2(+)-sensing, second messengers, and the control of parathyroid hormone secretion. Cell Calcium 11: 333-337, 1990.

8. Goldsmith PK, Fan GF, Ray K, et al: Expression, purification, and biochemical characterization of the amino-terminal extracellular domain of the human calcium receptor. J Biol Chem 274: 11303-11309, 1999

9. Hurley JB: Visual transduction. Sensing calcium in rod cells. Nature 361: 20-21, 1993.

10. Juhlin C, Lundgren S, Johansson $\mathrm{H}$, et al: 500-Kilodalton calcium sensor regulating cytoplasmic $\mathrm{Ca}^{2+}$ in cytotrophoblast cells of human placenta. J Biol Chem 265: 8275-8279, 1990.

11. Sin IL, Fernandes R and Mercola D: Direct identification of the high and low affinity calcium binding sites of troponin-C. Biochem Biophys Res Commun 82: 1132-1139, 1978.

12. Terasawa M, Nakano A, Kobayashi R and Hidaka H: Neurocalcin a novel calcium-binding protein from bovine brain. J Biol Chem 267: 19596-19599, 1992.

13. Kobayashi M, Takamatsu K, Saitoh S, Miura M and Noguchi T: Molecular cloning of hippocalcin, a novel calcium-binding protein of the recoverin family exclusively expressed in hippocampus. Biochem Biophys Res Commun 196: 1017, 1993.

14. Lenz SE, Henschel Y, Zopf D, Voss B and Gundelfinger ED: Vilip, a cognate protein of the retinal calcium-binding proteins visinin and recoverin, is expressed in the developing chicken brain. Mol Brain Res 15: 133-140, 1992.

15. Pertzev A, Duda T and Sharma RK: $\mathrm{Ca}(2+)$ sensor GCAP1: a constitutive element of the ONE-GC-modulated odorant signal transduction pathway. Biochemistry 49: 7303-7313, 2010.

16. Adato A, Michel V, Kikkawa $\mathrm{Y}$, et al: Interactions in the network of Usher syndrome type 1 proteins. Hum Mol Genet 14: 347-356, 2005.

17. Donaudy F, Snoeckx R, Pfister M, et al: Nonmuscle myosin heavy-chain gene MYH14 is expressed in cochlea and mutated in patients affected by autosomal dominant hearing impairment (DFNA4). Am J Hum Genet 74: 770-776, 2004.

18. Pusch CM, Meyer B, Kupka S, et al: Refinement of the DFNA4 locus to a $1.44 \mathrm{Mb}$ region in 19q13.33. J Mol Med 82: 398-402, 2004.

19. Bearer EL, Chen AF, Chen AH, et al: 2E4/Kaptin (KPTN)-a candidate gene for the hearing loss locus, DFNA4. Ann Hum Genet 64: 189-196, 2000.

20. Delprat B, Michel V, Goodyear R, et al: Myosin XVa and whirlin, two deafness gene products required for hair bundle growth, are located at the stereocilia tips and interact directly. Hum Mol Genet 14: 401-410, 2005.

21. Brose N, Petrenko AG, Sudhof TC and Jahn R: Synaptotagmin: a calcium sensor on the synaptic vesicle surface. Science 256 : 1021-1025, 1992.

22. Weber T, Zemelman BV, McNew JA, et al: SNAREpins: minimal machinery for membrane fusion. Cell 92: 759-772, 1998.

23. Safieddine $S$ and Wenthold RJ: SNARE complex at the ribbon synapses of cochlear hair cells: analysis of synaptic vesicle- and synaptic membrane-associated proteins. Eur J Neurosci 11: 803-812, 1999.

24. Sutton RB, Davletov BA, Berghuis AM, Sudhof TC and Sprang SR: Structure of the first $\mathrm{C} 2$ domain of synaptotagmin I: a nove $\mathrm{Ca}^{2+} /$ phospholipid-binding fold. Cell 80: 929-938, 1995
25. Yasunaga S, Grati M, Chardenoux S, et al: OTOF encodes multiple long and short isoforms: genetic evidence that the long ones underlie recessive deafness DFNB9. Am J Hum Genet 67: 591-600, 2000.

26. Therrien C, Di Fulvio S, Pickles S and Sinnreich M: Characterization of lipid binding specificities of dysferlin $\mathrm{C} 2$ domains reveals novel interactions with phosphoinositides. Biochemistry 48: 2377-2384, 2009

27. Roux I, Safieddine S, Nouvian R, et al: Otoferlin, defective in a human deafness form, is essential for exocytosis at the auditory ribbon synapse. Cell 127: 277-289, 2006.

28. Pangrsic T, Lasarow L, Reuter K, et al: Hearing requires otoferlindependent efficient replenishment of synaptic vesicles in hair cells. Nat Neurosci 13: 869-876, 2010.

29. Johnson CP and Chapman ER: Otoferlin is a calcium sensor that directly regulates SNARE-mediated membrane fusion. J Cell Biol 191: 187-197, 2010.

30. Schug N, Braig C, Zimmermann U, et al: Differential expression of otoferlin in brain, vestibular system, immature and mature cochlea of the rat. Eur J Neurosci 24: 3372-3380, 2006.

31. Heidrych P, Zimmermann U, Kuhn S, et al: Otoferlin interacts with myosin VI: implications for maintenance of the basolateral synaptic structure of the inner hair cell. Hum Mol Genet 18: 2779-2790, 2009

32. Heidrych P, Zimmermann U, Bress A, et al: Rab8b GTPase, a protein transport regulator, is an interacting partner of otoferlin, defective in a human autosomal recessive deafness form. Hum Mol Genet 17: 3814-3821, 2008.

33. Heidrych P: Novel interaction partners for Otoferlin, a functional member of acoustic transmission in the inner ear. University of Tuebingen, Tuebingen, 2008.

34. Bulgakov OV, Eggenschwiler JT, Hong DH, Anderson KV and $\mathrm{Li}$ T: FKBP8 is a negative regulator of mouse sonic hedgehog signaling in neural tissues. Development 131: 2149-2159, 2004.

35. Edlich F, Weiwad M, Wildemann D, et al: The specific FKBP38 inhibitor $\mathrm{N}-\left(\mathrm{N}^{\prime}, \mathrm{N}\right.$-dimethylcarboxamidomethyl)cycloheximide has potent neuroprotective and neurotrophic properties in brain ischemia. J Biol Chem 281: 14961-14970, 2006.

36. Shirane $M$ and Nakayama KI: Inherent calcineurin inhibitor FKBP38 targets $\mathrm{Bcl}-2$ to mitochondria and inhibits apoptosis. Nat Cell Biol 5: 28-37, 2003.

37. Portier BP and Taglialatela G: Bcl-2 localized at the nuclear compartment induces apoptosis after transient overexpression. J Biol Chem 281: 40493-40502, 2006.

38. Walker VE, Atanasiu R, Lam H and Shrier A: Co-chaperone FKBP38 promotes HERG trafficking. J Biol Chem 282: 23509-23516, 2007.

39. Okamoto T, Nishimura Y, Ichimura T, et al: Hepatitis $C$ virus RNA replication is regulated by FKBP8 and Hsp90. EMBO J 25: 5015-5025, 2006.

40. Bonifacino JS and Traub LM: Signals for sorting of transmembrane proteins to endosomes and lysosomes. Annu Rev Biochem 72: 395-447, 2003.

41. Buss F, Arden SD, Lindsay M, Luzio JP and Kendrick-Jones J: Myosin VI isoform localized to clathrin-coated vesicles with a role in clathrin-mediated endocytosis. EMBO J 20: 3676-3684, 2001.

42. Duncker SV: Identifiezierung von AP-2 und Cask als neue Interaktionspartner von Otoferlin bzw. Prestin in cochlearen Haarsinneszellen. University of Tuebingen, Tuebingen, 2010.

43. Brandt N, Kuhn S, Munkner S, et al: Thyroid hormone deficiency affects postnatal spiking activity and expression of $\mathrm{Ca}^{2+}$ and $\mathrm{K}^{+}$ channels in rodent inner hair cells. J Neurosci 27: 3174-3186, 2007.

44. Rodriguez-Ballesteros M, del Castillo FJ, Martin Y, et al: Auditory neuropathy in patients carrying mutations in the otoferlin gene (OTOF). Hum Mutat 22: 451-456, 2003 